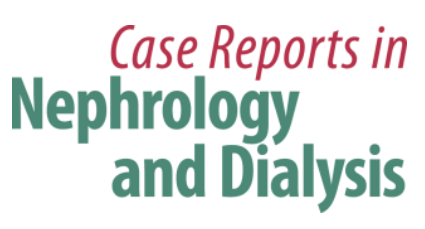

\title{
Acute Interstitial Nephritis and Membranous Nephropathy in the Context of IgG4-Related Disease
}

\author{
Kostas Stylianou $^{\mathrm{a}} \quad$ Eleftheria Maragkaki $^{\mathrm{a}}$ Michael Tzanakakis $^{\mathrm{a}}$ \\ Stavros Stratakis $^{a}$ Hariklia Gakiopoulou ${ }^{b}$ Eugene Daphnis ${ }^{a}$ \\ ${ }^{a}$ Nephrology Department, University Hospital of Heraklion, Heraklion, and ${ }^{b}$ Pathology \\ Department, National and Kapodistrian University of Athens, Athens, Greece
}

\section{Key Words}

Interstitial nephritis $\cdot$ IgG4-related disease $\cdot$ Membranous nephropathy

\begin{abstract}
We present the case of a patient with IgG4-related disease, which manifested in an asynchronous manner as vitiligo, cholecystitis, sialadenitis, lymphadenopathy, facial palsy and kidney dysfunction. The patient underwent a renal biopsy, and a presumptive diagnosis of lupus nephritis was made due to compatible clinical and immunological findings. The biopsy revealed IgG4-related kidney disease with severe interstitial nephritis and membranous nephropathy. Corticosteroids treatment restored all disease manifestations. We bring this case to the attention of the nephrologists because of the protean, asynchronous, multisystemic nature of the disease that necessitates a multidisciplinary approach, a low threshold for kidney biopsy and a high index of suspicion for making the correct diagnosis and treatment.
\end{abstract}

(C) 2014 S. Karger AG, Basel

\section{Background}

IgG4-related disease (IgG4-RD) is a protean systemic disease that can affect many organs such as the pancreas, gall bladder, liver, thyroid, lungs, kidneys, skin, cranial nerves, retroperitoneum, prostate, as well as pituitary, salivary and lacrimal glands [1]. Swelling and enlargement of the affected organ(s) with characteristic lymphoplasmacytic infiltrations and remarkably high serum IgG and IgG4 are the major diagnostic clues. Kidney involvement by tubulointerstitial nephritis (TIN) was first described in 2004 [2, 3]. Several other cases have been reported since then, justifying the term IgG4-related TIN proposed in 2010 [4]. 


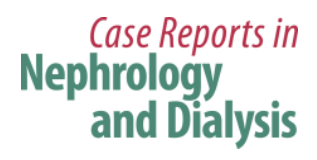

\begin{tabular}{l|l}
\hline Case Rep Nephrol Dial 2015;5:44-48 \\
\hline DOI: $10.1159 / 000369924$ & $\begin{array}{l}\text { C 2014 S. Karger AG, Basel } \\
\text { www.karger.com/cnd }\end{array}$ \\
\hline
\end{tabular}

Stylianou et al.: Acute Interstitial Nephritis and Membranous Nephropathy in the Context of IgG4-Related Disease

However, IgG4-RD has also been described in relation with glomerular lesions such as membranous nephropathy (MN) and other glomerulopathies with or without the presence of TIN, broadening the spectrum of kidney involvement to the term IgG-related kidney disease (IgG4-RKD). Here, we present the case of a patient with several clinical manifestations compatible with IgG4-RD, who remained undiagnosed for many years until his kidney function was compromised.

\section{Case Report}

A 63-year-old male was admitted to his regional hospital because of severe weakness, anorexia and weight loss (16 kg in the previous 4 months). Six months earlier, he had been admitted to the same hospital with fatigue, cervical lymphadenopathy, sialadenitis and peripheral facial palsy. He had then received oral steroids for 2 months with significant clinical improvement. Prior medical history included hypertension for 15 years, a selflimited vitiligo 2 years before and an episode of alithiasic cholecystitis, without pancreatic involvement 1 year before.

On admission, laboratory tests showed anemia (hemoglobin $8.2 \mathrm{~g} / \mathrm{dl}$ ), leukocytosis (WBCs $18.800 / \mathrm{ml}$ ) with increased neutrophils (60-88\%) and eosinophils (3-18\%), increased C-reactive protein, rapid deterioration of renal function (peak urea $87 \mathrm{mg} / \mathrm{dl}$, creatinine $2.8 \mathrm{mg} / \mathrm{dl}$ ), positive antinuclear antibodies (ANA) 1:1,280, low serum complement, hypoalbuminemia $(1.8 \mathrm{~g} / \mathrm{dl})$, proteinuria (1.8 g/daily), pyuria and mild hematuria in urinalysis. He was then referred to our hospital for renal biopsy with a presumptive diagnosis of lupus nephritis. On admission to our hospital, the patient had a low-grade fever, intense weakness affecting mobility, absence of arthralgias or arthritis. He was hemodynamically stable and normovolemic. The rest of the physical examination revealed only hepatomegaly and muscle wasting. Laboratory tests confirmed the inflammatory syndrome (elevated erythrocyte sedimentation rate and C-reactive protein) and immunological abnormalities with high IgG $(2,020 \mathrm{mg} / \mathrm{dl})$, low complement C3 and C4, positive ANA 1:640 and atypical pANCA 1:160. Antibodies against double-stranded DNA (anti-dsDNA) were found slightly positive only once, whereas the crithidia luciliae immunofluorescence (CLIF) test was negative. Serum and urine immunofixation, MPO/PR3 titers, hepatitis screening, serum cryoglobulins and angiotensin-converting enzyme were negative or normal. Both abdominal ultrasound and CT revealed large kidneys $(13$ and $14 \mathrm{~cm}$ ) and marked hepatomegaly. Brain CT was normal and chest CT showed marginally enlarged mediastinal lymph nodes. Further analysis of IgG subclasses revealed extremely high IgG4 levels $(1,210 \mathrm{mg} / \mathrm{dl})$. A subsequent kidney biopsy showed irregular thickening of glomerular basement membranes (GBMs) with C4d deposition by immunohistochemistry, thickening of tubular basement membranes (TBMs) and diffuse interstitial inflammatory infiltrates disrupting normal kidney architecture. The infiltrates consisted mainly of polyclonal (kappa and lambda positive) plasma cells, the vast majority being IgG4+ $>50$ per high power field, hpf) as well as CD3+ and CD20+ lymphocytes and eosinophils (fig. 1). Congo red stain was negative. Interstitial fibrosis was estimated at $30-35 \%$ of the substrate by Masson stain. Immunofluorescence showed moderate IgG and mild C3 depositions in GBMs. IgG was also found in interstitial infiltrates and IgM in the TBMs. Fluorescence for IgA, C4 and C1q was negative. Electron microscopy revealed the presence of several subepithelial dense deposits and scarce mesangial and TBM deposits. Based on biopsy findings, a final diagnosis of IgG4RKD with TIN and secondary MN was made. Methylprednisolone therapy was started at a dose of $36 \mathrm{mg} /$ day with gradual tapering to $4 \mathrm{mg} /$ day for 1 year. This treatment resulted in a 
Stylianou et al.: Acute Interstitial Nephritis and Membranous Nephropathy in the Context of IgG4-Related Disease

rapid improvement of symptoms and renal function (serum creatinine from 2.8 to $1.8 \mathrm{mg} / \mathrm{dl}$ within the first 10 days). At the 6 and 12 months follow-up, clinical, radiologic and laboratory findings have all returned to normal (serum creatinine at $1.1 \mathrm{mg} / \mathrm{dl}$ ).

\section{Discussion}

IgG4-RD mainly affects middle-aged and elderly men, who frequently present allergic diathesis. Many organs can be affected either synchronously or asynchronously. The most common renal manifestation is plasma cell-rich TIN with 'storiform' fibrosis and dense deposits in the TBM. More specific criteria that have been proposed for the diagnosis of IgG4-RKD include: (a) elevated serum total IgG levels or IgG4 (>135 mg/dl); (b) plasma cellrich TIN with an absolute number of IgG4+ plasma cells $>10$ per hpf, obliterative phlebitis and storiform fibrosis; (c) kidney enlargement with wedge-shaped or patchy lesions in imaging studies, and (d) characteristic findings of IgG4-RD in other organs [5-7]. Hypocomplementemia and high serum IgE levels are also evident in 70\% of patients [8]. Occasionally, glomerular lesions have also been described with MN being the most frequent $7 \%$ of patients with IgG4-RKD), with or without TIN. Anti-M-type phospholipase A2 receptor antibodies were negative in all such cases [9]. Since no single test can definitively diagnose IgG4-RD, we rely on a combination of histologic, clinical, radiographic, and laboratory findings. Diagnosis of IgG4-TIN requires the histologic feature of IgG4 plasma cell-rich TIN and at least one other feature from the imaging, serology, or other organ involvement categories [7].

In almost $70 \%$ of cases, the disease may mimic systemic lupus erythematosus with positive ANA and low complement levels, whereas anti-dsDNA, rheumatoid factor, SSA, SSB and ANCA are negative [6]. This particular patient displayed an asynchronous multisystemic disease resembling systemic lupus erythematosus. However, the very specific CLIF test was negative, subsequent anti-dsDNA titers were negative, myeloperoxidase titers were normal by ELISA, and kidney histology excluded the diagnosis of vasculitis or lupus. On the contrary, biopsy findings were highly suggestive of IgG4-RKD based on the 2012 consensus statement on the pathology of IgG4-RD [10]. In particular, although there was no evidence of obliterative phlebitis, there were dense lymphoplasmacytic infiltrations with >50 IgG4+ plasma cells per hpf, eosinophilic infiltrates and storiform fibrosis. Furthermore, in agreement with the existing literature $[6,8]$, the patient dramatically responded to steroid treatment with regard to all aspects of his IgG4-RD. Therefore, we consider positive anti-dsDNA and ANCA as nonspecific and rather confusing findings. Skin lesions in patients with IgG4-RD usually consist of erythematous and itchy plaques or subcutaneous nodules on the skin of the head and neck [11]. However, we consider that the previous history of self-limited vitiligo, another autoimmune disorder, may be part of the clinical spectrum of IgG4-RD since it has been reported in 2 more patients with IgG4-RD $[12,13]$. The protean, multisystemic, nature of the disease necessitates a multidisciplinary approach, a low threshold for tissue biopsy and a high index of suspicion for making the correct diagnosis. Early diagnosis and treatment can completely reverse kidney dysfunction.

\section{Disclosure Statement}

All authors declare that no conflicts of interest exist and an informed consent has been obtained from the patient. 
Stylianou et al:: Acute Interstitial Nephritis and Membranous Nephropathy in the Context of IgG4-Related Disease

\section{References}

1 Fragoulis GE, Moutsopoulos HM: IgG4 syndrome: old disease, new perspective. J Rheumatol 2010;37:13691370.

-2 Uchiyama-Tanaka Y, Mori Y, Kimura T, Sonomura K, Umemura S, Kishimoto N, et al: Acute tubulointerstitial nephritis associated with autoimmune-related pancreatitis. Am J Kidney Dis 2004;43:e18-e25.

-3 Takeda S, Haratake J, Kasai T, Takaeda C, Takazakura E: IgG4-associated idiopathic tubulointerstitial nephritis complicating autoimmune pancreatitis. Nephrol Dial Transplant 2004;19:474-476.

4 Saeki T, Nishi S, Imai N, Ito T, Yamazaki H, Kawano M, et al: Clinicopathological characteristics of patients with IgG4-related tubulointerstitial nephritis. Kidney Int 2010;78:1016-1023.

-5 Umehara H, Okazaki K, Masaki Y, Kawano M, Yamamoto M, Saeki T, et al: Comprehensive diagnostic criteria for IgG4-related disease (IgG4-RD), 2011. Mod Rheumatol 2012;22:21-30.

6 Saeki T, Kawano M: IgG4-related kidney disease. Kidney Int 2014;85:251-257.

7 Raissian Y, Nasr SH, Larsen CP, Colvin RB, Smyrk TC, Takahashi N, et al: Diagnosis of IgG4-related tubulointerstitial nephritis. J Am Soc Nephrol 2011;22:1343-1352.

-8 Saeki T, Kawano M, Mizushima I, Yamamoto M, Wada Y, Nakashima H, et al: The clinical course of patients with IgG4-related kidney disease. Kidney Int 2013;84:826-833.

-9 Alexander MP, Larsen CP, Gibson IW, Nasr SH, Sethi S, Fidler ME, et al: Membranous glomerulonephritis is a manifestation of IgG4-related disease. Kidney Int 2013;83:455-462.

10 Deshpande V, Zen Y, Chan JK, Yi EE, Sato Y, Yoshino T, et al: Consensus statement on the pathology of IgG4related disease. Mod Pathol 2012;25:1181-1192.

-11 Sato Y, Takeuchi M, Takata K, Ohno K, Iwaki N, Orita Y, et al: Clinicopathologic analysis of IgG4-related skin disease. Mod Pathol 2013;26:523-532.

12 Bourlon MT, Sánchez-Ávila M, Chablé-Montero F, Arceo-Olaiz R: IgG4-related autoimmune prostatitis: is it an unusual or underdiagnosed manifestation of IgG4-related disease? Case Rep Urol 2013;2013:295472.

-13 Hermet M, André M, Kémény JL, Le Guenno G, Déchelotte P, Guettrot-Imbert G, et al: Is IgG4-related disease a cause of xerostomia? A Cohort study of 60 patients. Int J Rheumatol 2012;2012:303506.

The results presented in this paper have not been published previously in whole or part, except in abstract format. 


\section{Case Reports in \\ Nephrology and Dialysis}

\begin{tabular}{l|l}
\hline Case Rep Nephrol Dial 2015;5:44-48 \\
\hline DOI: 10.1159/000369924 & $\begin{array}{l}\text { ○ 2014 S. Karger AG, Basel } \\
\text { www.karger.com/cnd }\end{array}$ \\
\hline
\end{tabular}

Stylianou et al.: Acute Interstitial Nephritis and Membranous Nephropathy in the Context of IgG4-Related Disease
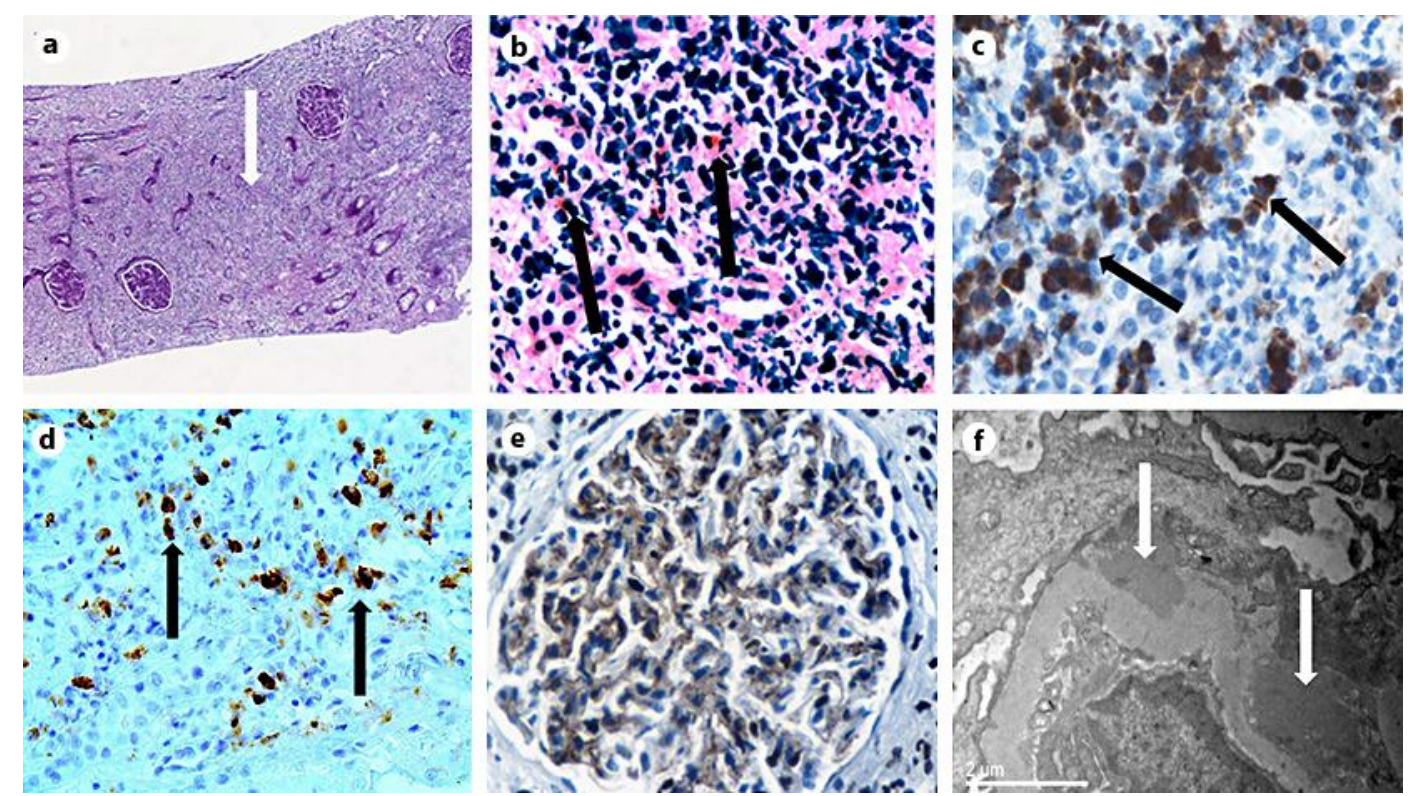

Fig. 1. a Rarefaction of tubules and glomeruli, severe interstitial inflammatory infiltrates and fibrosis (arrow), PAS $\times 10$. b Interstitial nephritis with dense lymphoplasmacytic infiltrations and eosinophils (arrows), PAS $\times 400$. c The majority of interstitial inflammatory infiltrates were IgG-producing plasma cells (arrows). Immunohistochemistry for IgG, $\times 400$. $\mathbf{d}$ The majority $(>40 \%)$ of IgG+ plasma cells appear positive for IgG4 (arrows). In absolute numbers, $>50$ IgG4+ plasma cells are seen per hpf. Immunohistochemistry for IgG4, $\times 400$. e Focal thickening of GBMs with C4d positivity indicating MN lesions. Immunohistochemistry for $\mathrm{C} 4 \mathrm{~d}, \times 200$. $\mathrm{f}$ Electron microscopy revealed the presence of subepithelial (arrows) and scarce mesangial deposits compatible with secondary MN, $\times 15.000$. 